\title{
Educação alimentar e nutricional, cultura e subjetividades: a escola contribuindo para a formação de sujeitos críticos e criativos em torno da cultura alimentar
}

\section{Nutritional food education, culture and subjectivities: school contributing to the formation of critical and creative people around food culture}

\author{
Antonio Carlos Barbosa da Silva' \\ Marina Coimbra Casadei Barbosa \\ da Silva ${ }^{2}$ \\ Vanessa Érika Rodrigues de Oliveira ${ }^{3}$ \\ 1 Universidade Estadual Paulista "Júlio de \\ Mesquita Filho", Departamento de Psicologia \\ Evolutiva, Social e Escolar. Assis-SP, Brasil. \\ 2 Universidade Estadual Paulista "Júlio de \\ Mesquita Filho", Faculdade de Filosofia e \\ Ciências, Pós-graduação em Educação. Marília- \\ SP, Brasil. \\ ${ }^{3}$ Etec José Martimiano da Silva. Ribeirão \\ Preto-SP, Brasil. \\ 0 presente trabalho foi subvencionado pelo \\ CNPq/MDS/Sesan - Processo 407514/2012-0 \\ Correspondência / Correspondence \\ Antonio Carlos Barbosa da Silva \\ E-mail: tom@assis.unesp.br
}

\section{Resumo}

A partir de uma série de intervenções grupais, construímos, com um grupo de estudantes da rede pública de ensino, reflexões em torno da educação e da cultura alimentar e nutricional em vigor na sociedade contemporânea. Utilizando como base teórica para as reflexões a teoria histórico-cultural e a história da alimentação, debatemos com os estudantes quais elementos históricos, sociais, políticos, ideológicos e econômicos influenciam a cultura alimentar e como interferem na formação da subjetividade do sujeito. Concluímos que o debate em torno dos hábitos alimentares tradicionais tem o mérito de resgatar a cultura e história da alimentação, e de como ela é extremamente relevante na formação da identidade cultural e social de um povo. Ações em educação alimentar e nutricional podem potencializar a criticidade de estudantes a respeito dos hábitos alimentares, que na maioria das vezes são impostos por estratégias mediáticas da indústria alimentar, e conduzi-los a rever a relação que estabelecem com a alimentação na contemporaneidade.

Palavras-chave: Educação. Alimentação. Hábitos alimentares. Indústria Alimentícia. Psicologia. Cultura.

\section{Abstract}

From a series of group interventions we built, with a group of students from public schools, reflections on education and nutrition and food culture in contemporary society. Using as theoretical basis for reflections the historical-cultural theory and history of food, we debated with students which historical, social, political, ideological and economic factors influence food 
culture and how to interfere in the formation of the subjectivity of the subject. We conclude that the debate on traditional eating habits has the merit of rescuing the culture and history of food, and how it is extremely relevant in the formation of cultural and social identity of a people. Actions in food and nutrition education can enhance the criticality of students regarding eating habits, which most often are imposed by media strategies of the food industry, and lead them to review the relationship they establish with food nowadays.

Key words: Education. Food. Food Habits. Food Industry Psychology. Culture.

\section{Introdução}

\section{Cultura alimentar e história}

A cultura e a história são criações sociais do saber humano, do fazer humano e do criar humano. Dessa forma, o que sentimos, pensamos, falamos e criamos são momentos do trabalho criativo da realização da pessoa humana no contexto de uma cultura.

Ao longo da construção desses elementos, estão presentes experiências, hábitos, atitudes, valores, a linguagem de outros indivíduos com quem o sujeito se relaciona e de outras instituições próximas a ele, como, por exemplo, a escola, a igreja, os grupos familiares etc. Assim, a história e a cultura dos antepassados, próximos e distantes, são peças importantes na construção do desenvolvimento humano.

Entretanto, esse processo não é um determinismo histórico e cultural em que, passivamente, o sujeito absorve certos comportamentos para reproduzi-los posteriormente. Ele deve participar ativamente da construção de sua própria cultura e de sua história, modificando-se e provocando transformações nos demais sujeitos e instituições que com ele interagem.

A comida seria um elemento histórico-cultural, que iria além do sentido de nutrir-se para sobreviver. Ela estaria relacionada aos aspectos pessoais, subjetivos, familiares e históricos presentes na preparação e no consumo dos alimentos. Na alimentação, estariam contidos aspectos culturais de determinada sociedade, comunidade e religião. 
De acordo com Carneiro, ${ }^{1}$ a comida representa, portanto, um ato da cultura material de uma sociedade, fazendo parte de sua infraestrutura e de sua superestrutura social - ou seja, a alimentação é um fato ideológico, das representações religiosas, artísticas e morais da sociedade. ${ }^{1}$ É uma das necessidades básicas do homem, e foi buscando aplacar essa necessidade que os homens se organizaram, criando, assim, a cultura. Nesse sentido, os processos que envolvem a alimentação foram utilizados como estratégias fundamentais para a comunhão e organização social dos homens.

Para Carvalho, ${ }^{2}$ a comida representou uma riqueza para o homem e foi capaz de expressar, ao longo de sua história, a constituição das estruturas sociais que o diferenciavam de outros animais. A comida envolveria, então, organização (preparação para caça ou preparação para ao plantio), trabalho (ato de caçar e coletar) e divisão de tarefas (quem prepararia o fogo, quem limparia, quem cozinharia o alimento). As questões dessa divisão (que são sociais) vão desde como conseguir o alimento, plantio ou extrativismo, caça ou criação, até de quem vai se ocupar do plantio e do preparo, papéis masculinos e femininos que reproduzimos, de certa maneira, até hoje nos cuidados domésticos com alimentação e educação nos núcleos familiares.

Era no cozimento que prevalecia a arte de elaborar os alimentos, dar-lhes sabor e sentido e torná-los elementos da cultura daquele grupo. Era no ato de comer que fortalecíamos a intimidade familiar e a afetividade, e nele despontavam as relações de gênero, gerações, emoções e atividades que traduziam a relação do homem no mundo e com o mundo ${ }^{3}$.

O fato de nos reunirmos para preparar certas comidas nos levava a entrar em contato com os elementos culturais regionais que envolviam a comida e seu preparo, tais como as escolhas de determinados temperos, o tipo de corte dos alimentos, o desprezo de algumas partes do alimento etc. Diante disso, verificamos que a cultura alimentar tem certo patrimônio e é resultado de um longo processo de aprendizagem que se inicia no momento do nascimento e se consolida no contexto familiar e social.

Por essa razão, é fácil compreender que as diferenças na alimentação podem ser vividas com uma mescla de surpresa, estranheza, desconfiança e, em certos casos, repulsa. As formas de alimentação, os produtos consumidos e a forma de cozinhá-los relacionam- se com as peculiaridades de cada povo, tais como os recursos locais, as características do clima e dos solos - ou seja, com o território, as formas de produção, a agricultura, a pecuária, suas formas de armazenamento e o comércio. ${ }^{4}$

Como afirma Zunin, ${ }^{5}$ esse conjunto de elementos e tudo a eles relacionado são percebidos como "marcadores étnicos". Noutros termos, a alimentação é um dos elementos que contribuem para "gerar elementos identitários do sujeito", mediante a constatação da diferença alimentar. 
De um modo geral, a alimentação e as práticas culinárias estão inscritas em um contexto antropológico e socioeconômico determinado. Para Carvalho, ${ }^{2}$ se a comida era ela mesma uma riqueza cultural, com o passar do tempo, novos materiais e novas técnicas foram incorporados, permitindo acumular excedentes de produção alimentícia. A comida pôde então ser trocada, assumindo valores e preços, expressando, assim, um processo de transformação social do alimento.

Na era contemporânea, a comida parece sofrer as imposições de um campo de força que tem no modelo da globalização e do neoliberalismo seus exemplos. Assim, o alimento que pode ser reproduzido exatamente em formato, sabor, textura e cor nas mais diferentes e longínquas regiões do planeta, e o alimento feito do modo mais rápido, que proporciona prazer e adequação nutricional, serão os mais valorizados.

Portanto, o mundo atual, marcado pela velocidade, pela produção excessiva e sobressalente de produtos, não valoriza os rituais que envolvem o ato de comer. A falta de tempo levou o sistema neoliberal a desenvolver todo um mercado de alimentos semiprontos, congelados, práticos, individualizantes etc. $\mathrm{O}$ mundo contemporâneo não permite que gastemos nosso tempo em "futilidades" como escolher, limpar, preparar o alimento e sentar na mesa, e nela perder tempo comendo e conversando sem grandes objetividades.

\section{A cultura alimentar na contemporaneidade}

De certo modo, a indústria alimentícia é um esclarecedor exemplo de como a organização capitalista modifica hábitos alimentares culturais e finda por minimizar a cultura alimentar de um povo. Os brasileiros, diante dos encontros de suas três matrizes étnicas (indígena, europeia e africana), desenvolveram uma rica cultura alimentar. Entretanto, diante das mazelas do mundo contemporâneo, perdem-na a cada dia. Os alimentos ultraprocessados aportam na mesa dos brasileiros e a comida dos fast foods tomam o lugar da feitura e do compartilhamento da mesa dos almoços familiares do dia a dia.

Não foi fortuito o crescente sucesso das cadeias alimentares de fast food, abordadas por Rial, ${ }^{6}$ que muito investiram na publicidade alimentar e, pela via das imagens, veicularam novas representações sobre o modo de vida contemporâneo, evidenciando muito mais a operacionalização, rapidez na confecção do alimento e a padronização do sabor da comida.

Rial $^{6}$ ressaltou a especificidade da culinária do fast food na divulgação de novas formas de se alimentar, na redefinição dos espaços das refeições e do seu tempo, junto com a modificação da própria estrutura da alimentação. No passado, a alimentação era demarcada geográfica, temporal e simbolicamente, e as ocorrências alimentares separavam o tempo, estimulando a sociabilidade familiar, ou interrompiam a jornada de trabalho, marcando os momentos cotidianos e não 
cotidianos. As formas de alimentação no mundo moderno e as mudanças nas práticas alimentares movem-se pela demarcação de novos espaços e velocidade.

De um modo geral, a alimentação representaria a manifestação da formação social e cultural de um povo, a chave simbólica dos costumes, um código subjetivo que registraria, forjaria identidades, denunciaria e evidenciaria um modo de pensar e sentir o mundo.

As observações sobre os diversos elementos simbólicos que cerceariam os alimentos facilitariam a interpretação de conceitos construídos por diferentes sujeitos sociais, justificando seus hábitos alimentares e suas articulações concretas do cotidiano, enquanto estratégias de vida. Essas estratégias deveriam ser pontuadas pela EAN (educação alimentar e nutricional) porque simbolizariam os processos subjetivos que estariam por detrás da escolha de certos alimentos em detrimento de outros, evidenciariam culturas e resgatariam a história do indivíduo e sua comunidade. Em suma, a educação alimentar e nutricional poderia fornecer elementos aos sujeitos que poderiam torná-los conhecedores de sua cultura alimentar e da cultura de seu povo.

Assim, a partir dessas considerações, poderíamos associar alguns aspectos da alimentação aos fatores culturais, manifestando-se através do consumo consciente ou inconsciente do alimento, o que pode incorrer na má nutrição. Os importantes papéis culturais que os alimentos desempenhariam nas sociedades fariam parte das tentativas de modificar ou aperfeiçoar os hábitos alimentares dos indivíduos.

O consumo de certos alimentos na contemporaneidade também revela aspectos sociais. Quando os alimentos são consumidos por um grupo de pessoas e são dotados de valor simbólico representariam status social, na medida em que a qualidade e quantidade da comida poderiam refletir o poder aquisitivo dos indivíduos.

No mundo contemporâneo, desenvolve-se junto à elite um estilo de vida gourmet (comidas prazerosas, raras e nutritivas, feitas sob medida para o sujeito), e junto às camadas mais carentes, uma alimentação meramente calórica, não importando muito de onde vêm essas calorias, que servem para abrandar a fome e energizar o indivíduo. Portanto, vemos que o alimento possui conotações econômicas, sociais e culturais, podendo afetar a nutrição dos indivíduos ao excluir nutrientes essenciais da alimentação ou estimulando o consumo de determinados alimentos ou bebidas.

De fato, se invertemos o ponto de vista social de referência e passamos do contexto da pobreza ao da riqueza, o mecanismo de formação do gosto parece, também ele, inverter-se. Objeto e desejo não é mais o alimento abundante, mas o raro; não aquele que enche e faz passar a fome, mas aquele que estimula e convida a comer mais. ${ }^{7}$

Hoje em dia, a industrialização e a tecnologia possibilitaram a produção e o barateamento em excesso de produtos alimentícios, mas esses produtos, disfuncionais e hipercalóricos, são destinados 
a uma parcela da população que dispõe de parcos recursos financeiros, enquanto os alimentos funcionais, gourmet, saudáveis, são destinados às classes sociais mais abastadas.

\section{Educação Alimentar e Nutricional}

A partir da década de 1990, pesquisas científicas apontaram que os aumentos de várias doenças crônicas degenerativas estariam relacionados aos hábitos alimentares da população. Isso abriu caminhos para a instalação de políticas públicas que postularam a educação alimentar e nutricional (EAN) como medida necessária para a melhoria da saúde populacional.

Rocha $^{8}$ indicou que a Política Nacional de Alimentação e Nutrição (PNAN) e a EAN foram parâmetros factuais para a promoção da alimentação saudável, mas não se aprofundaram na reflexão, nem estabeleceram normas para promover uma educação crítica e cultural em torno da alimentação.

Para Boog, ${ }^{9}$ a promoção da EAN deveria valorizar e resgatar elementos da história e da cultura alimentar, considerando a segurança alimentar, respeitando e modificando crenças e atitudes em relação à alimentação. Seria também preciso levar em conta o acesso econômico e social da população à alimentação adequada, promovendo essa questão em programas comunitários, escolares e de saúde.

Outros componentes pertencentes ao contexto cultural, social e ecológico que envolveriam a alimentação deveriam ser também analisados, fazendo com que crenças e valores da população em relação à nutrição fossem reconhecidos, respeitados, refletidos e aprofundados e, quiçá, transformados. Assim, a alimentação e a atividade de se alimentar deveriam ser vistas como questões histórico-culturais, ultrapassando o sentido de nutrir-se para sobreviver, uma vez que nelas estariam contidos a história e aspectos culturais de determinada sociedade, comunidade e região - por exemplo, rituais, tipos específicos de consumo de alimentos (geralmente relacionados a preceitos de uma religião), diferentes modos de manufatura e produção, entre muitos outros.

Para Valente, ${ }^{10}$ ao considerarmos os processos históricos e sociais que estão por trás da nutrição e o possível fortalecimento dos movimentos populares na reivindicação de uma alimentação mais crítica e saudável, poderíamos estar criando um tipo de ação mais eficaz sobre a realidade da alimentação brasileira. Portanto, a EAN mais crítica desempenharia um papel importante ao educar os sujeitos na escolha de seus alimentos.

Na alimentação humana, natureza e cultura deveriam se encontrar, pois comer é uma necessidade vital, e quando e com quem comer são aspectos que fazem parte de um sistema que implicaria atribuição de significados ao ato alimentar. Como um fenômeno social, a alimentação não se restringiria a ser uma resposta ao imperativo de sobrevivência, ao "comer para viver", pois 
se os homens necessitariam sobreviver (e, para isso, alimentar-se), eles sobreviveriam de maneira particular, culturalmente forjada e marcada. Ou seja, os homens criam "maneiras de viver" diferentes, o que resultaria em grande diversidade cultural.

Cabe, portanto, aos educadores, estabelecer um diálogo junto à comunidade, a fim de mostrar que a alimentação tem elementos que iriam além da simples ingestão de alimentos. O comer envolveria respeito, regras, relações sociais, comensalidades e diversão. Em suma, comer envolveria cultura e história que seriam colocadas sobre a boa mesa.

\section{Objetivos}

Desenvolver ações em educação alimentar e nutricional junto ao um grupo de alunos do ensino médio de uma escola pública estadual, com a intenção de investigar os elementos históricos, culturais, midiáticos e ideológicos que estariam presentes na alimentação, e questionar o consumo excessivo de alimentos na forma de produtos industrializados ultraprocessados de caráter global.

\section{Método}

Nossa ação foi estruturada metodologicamente, de forma a construir espaços reflexivos na escola que proporcionam aos alunos pensar sobre a alimentação contemporânea, considerando elementos históricos e críticos a respeito da cultura alimentar. Para isso, fizemos uso das intervenções grupais em psicologia, com discussões junto aos alunos sob coordenação dos estagiários da pesquisa, com respaldo teórico da psicologia institucional, social comunitária e da pedagogia libertadora.

Documentos correspondentes à aprovação do comitê de ética da pesquisa foram apresentados aos responsáveis pela escola, que nos apoiaram no sentido de solicitar a autorização dos pais para realizar as atividades com estudantes. Nossa ação junto aos alunos se deu através da elaboração de duas oficinas de intervenções psicossociais relacionadas ao tema pesquisado - educação alimentar e cultura alimentar brasileira -, realizadas aos sábados pela manhã, junto às demais atividades da Escola da Família.

Na primeira oficina, contamos com 17 estudantes, entre 12 e 16 anos, de ambos os sexos. Nela desenvolvemos uma série de atividades psicológicas (dinâmicas de grupos, grupos de reflexão, debates em torno de trechos de documentários a respeito da cultura alimentar), coordenadas por bolsistas e orientadas pelo coordenador do projeto e pela pesquisadora responsável.

A reflexão em torno dos tipos de alimentos que consumíamos e como eram consumidos em períodos históricos anteriores foi uma tônica nos trabalhos. Também foram abordadas questões econômicas de ordem global que potencializam a indústria alimentar e modificam nossos hábitos 
modificando nossa cultura alimentar. Dessa forma, debatemos com os alunos temas como alimentos atualmente vendidos em supermercados. Ressaltamos também a importância das comidas que podem ser feitas de forma prazerosa, possibilitando o resgate de processos subjetivos esquecidos, tais como encontros familiares em torno dos alimentos e da alquimia dos temperos que nos fornecem novos sabores.

A segunda oficina foi desenvolvida com 15 estudantes com idade entre 12 a 16 anos, sendo meninos e meninas, muitos dos quais também participaram da primeira oficina. A oficina foi elaborada com o intuito de repensarmos nossa alimentação a partir de atividades práticas. Nesta atividade, contamos com o arsenal dos utensílios e materiais culinários fornecidos pela cozinha da escola, para refazermos algumas receitas da culinária brasileira. Ao resgatarmos as discussões históricas e sociais em torno da alimentação do brasileiro, durante o processo de feitura das receitas, podemos debater sobre o uso da farinha de mandioca, que além de fazer receitas saborosas, pode fornecer energia necessária para a resistência às intempéries de nossas regiões e lutas. Conversamos sobre o cupuaçu e o açaí, que são alimentos tão presentes no Sul e Sudeste, tão frequentes no Norte e preparados de modos diferentes em cada região. Também houve debates sobre comidas comuns e saborosas de todo o país, capazes de nos definir enquanto um povo, como o feijão com arroz.

\section{Resultados e conclusão}

As principais discussões junto aos grupos apontaram que as características dos modos de vida contemporâneos influenciaram significativamente o comportamento alimentar do jovem. Diante das amplas ofertas de opções dos alimentos processados e preparações alimentares semiprontas, além do apelo midiático, influência do marketing e da tecnologia de alimentos, o indivíduo estaria perdendo o contato social e coletivo das antigas formas ritualísticas que envolvem a preparação do alimento. A comida se tornou um objeto de consumo padronizado, a ser retirado das prateleiras do supermercado - basta tirá-la de sua embalagem e esquentá-la.

Outra questão que chamou muita atenção do grupo e que mereceu várias considerações foi sobre as patologias contemporâneas que surgem a partir de uma alimentação excessiva, com destaque para a obesidade.

Foi debatido, junto ao grupo, que na sociedade contemporânea o consumo excessivo de alimentos supérfluos culmina no sobrepeso e na obesidade de parte da população. A alimentação excessiva, em especial de produtos industrializados ultraprocessados, vem contribuindo para que as doenças crônicas degenerativas sejam uma das principais causas de morbidade e mortalidade no país. Em todas as classes sociais, houve aumento do consumo de produtos supérfluos e disfuncionais, decorrente da diminuição de preços, da tecnologia que aumentou a produção e de um agressivo marketing que não preservou nem as crianças. 
Em nossas ações, mostramos que a EAN seria um dos caminhos existentes para a promoção da saúde, para a reflexão sobre o comportamento alimentar e sobre a conscientização de uma alimentação para a saúde.

O debate em torno do reestabelecimento das refeições sociais junto às pessoas próximas também mereceu destaque. $\mathrm{O}$ ato de comer junto, o compartilhamento da refeição fortalece as relações sociais que outrora eram sociabilizadas em torno da comunhão da mesa e, atualmente, seriam rejeitadas em razão das refeições individualizadas, industrializadas e rápidas que faríamos em nosso dia a dia para atender às demandas de uma sociedade célere e hiperindividualista. A concepção de aprendizagem baseada nessa ação partiu da ideia de que o educando seria um sujeito ativo e que muitas de suas experiências e vivências poderiam surgir ao dividir a mesa e, consequentemente, estabeleceria diálogos com outros sujeitos mais experientes.

Segundo Boff, ${ }^{11}$ na mesa se faziam e se refaziam continuamente as relações familiares. A mesa, antes mais que um móvel, remeteria a uma experiência existencial, a um rito. Ela representaria lugar privilegiado da família, da comunhão e da irmandade. Partilhar-se-ia o alimento, e junto com ele viriam a alegria do encontro, o bem-estar sem disfarces, a comunhão direta que se traduziria pela não cerimônia dos comentários dos fatos cotidianos, das opiniões sem censura dos acontecimentos da história local, nacional e internacional. À mesa, além dos familiares, poderiam estar os amigos e os hóspedes. Seria à mesa que todos nos sentiríamos, de certa forma, membros da família humana.

Dessa forma, através de nossas ações, procuramos mostrar sempre a importância do diálogo nas oficinas desenvolvidas. De acordo com Freire, ${ }^{12}$ o diálogo também pode ser problematizado. Essa denominação dada pelo autor se deve à tarefa que cabe ao educador desenvolver, isto é, fazer com que o educando reflita e pense criticamente a respeito das coisas que estão a seu redor. Ao fazer o educando pensar criticamente, o educador cria a possibilidade para a produção e construção do conhecimento.

\section{Conclusões}

Educar no âmbito da alimentação e nutrição deveria visar uma construção conjunta de processos permanentes e contínuos para aprimorar o debate em torno da seleção e o consumo de alimentos, de forma adequada, saudável e segura. A valorização de hábitos e tradições culturais de cada indivíduo e do seu grupo social de convívio, além da conscientização cidadã sobre o desperdício de alimentos e sua utilização integral, deveria estar presente em propostas educativas em alimentação e nutrição. 
Estar atento para estabelecer diálogos consistentes, profundos e reveladores deveria ser a premissa de uma ação em EAN. Para Vigotsky, ${ }^{13}$ a linguagem agiria decisivamente na estrutura do pensamento e seria ferramenta básica para a construção de conhecimentos.

Portanto, desconstruir os saberes que o grupo traria em relação a sua alimentação, reconstruílos a par de novas considerações (sobretudo aquelas que seriam ocultas pela indústria marqueteira e alimentícia) e, concomitantemente, resgatar a cultura alimentar ignorada pelo grupo, mas constituinte do mesmo, foram peças fundamentais que fortaleceram nossa ação.

Com o atual desenvolvimento econômico de nosso país e o processo de globalização, que expõe todo tipo de mercadoria a uma política de marketing que oculta informações e valoriza predominantemente a estética, há um excesso do consumo de alimentos supérfluos, culminado em sobrepeso e obesidade de parte da população. Esse tipo de alimentação teria contribuído para que as doenças crônicas degenerativas fossem uma das principais causas de morbidade e mortalidade no país, em todas as classes sociais.

Dessa forma, a presente pesquisa-ação em EAN contribuiu para a efetivação de propostas intervencionistas que resgatem e se aprofundem nos elementos culturais e sociais que permeiam a nutrição e alimentação do brasileiro. Além disso, o debate em torno dos hábitos alimentares adquirido na contemporaneidade serve para resguardarmos os sujeitos de elementos críticos que os capacitaram para lidar contra a cultura de massa e a indústria alimentícia capitalista.

Até o momento, nossa pesquisa-ação teve o mérito de resgatar a cultura e história de nossa comida, e de como ela foi extremamente relevante para formar a identidade brasileira. Tal resgate possibilitou o desenvolvimento de um arsenal crítico e ativo do sujeito. Desta forma, o não preparo dos alimentos, juntamente com os familiares, consagrando as receitas e histórias de gerações, faria com que a memória histórica de um grupo e de gerações não fosse mantida, uma vez que a tradição também se relacionaria a memórias, recordações e costumes. Resgatar a tradição construída e transmitida há diversas gerações, responsável pela comunhão entre os homens, pelo respeito ao outro como humano, pela transmissão de valores morais que estão sendo perdidos, ou que já foram perdidos em diversas instâncias da vida cultural e social, aquela tradição cheia de ritos, ao redor da mesa na qual se realizaria comensalidade, seria uma das referências mais fundamentais da familiaridade humana que merece ser debatida e defendida em uma EAN.

Temas que relacionavam a alimentação à tradição, aos rituais, costumes, aos nutrientes e tabus, foram abordados em nossa EAN. Dessa maneira, "transmitir" valores e rituais históricos por meio de situações de ensino e aprendizagem fortaleceu a cultura alimentar dos estudantes.

A ação educativa em EAN possibilitou a reflexão sobre elementos culturais e ideológicos que envolvem a alimentação. Além disso, verificou que relações sociais que outrora eram sociabilizadas 
em torno da comunhão da mesa são atualmente rejeitadas em razão das refeições individualizadas, industrializadas e rápidas que serviram para atender às demandas de uma sociedade célere e hiperindividualista.

\section{Referências}

1. Carneiro H. Comida e sociedade: uma história da alimentação. Rio de Janeiro: Campus; 2003.

2. Carvalho MCVS, Luz MT, Prado, SD. Comer, alimentar e nutrir: categorias analíticas instrumentais no campo da pesquisa científica. Ciênc. Saúde Coletiva 2011; 16(1):155-163.

3. Zuin LFS, Zuin PB. Alimentação é cultura: aspectos históricos e culturais que envolvem a alimentação e o ato de se alimentar. Nutrire. Revista da Sociedade Brasileira de Alimentação e Nutrição. 2009; 34(1):225-241.

4. Contreras J. Patrimônio e globalização: o caso das culturas alimentares. In: Garcia RW. Antropologia e nutrição: um diálogo possível. Rio de Janeiro: Fiocruz; 2005. p. 129-146.

5. Zuin LFS, Zuin PB. Produção de alimentos tradicionais: extensão rural. Aparecida: Ideias \& Letras; 2008. 219 p.

6. Rial CSM. Os fast-foods: uma homogeneidade contestável na globalização cultural. Horizontes Antropológicos 1997; 5(1):140-180.

7. Montanari M. Comida como cultura. São Paulo: Senac; 2013. 207 p.

8. Rocha NC. Análise de programas de segurança alimentar e nutricional do Município de São Paulo [dissertação]. São Paulo: USP, Faculdade de Saúde Pública; 2011.

9. Boog MCF. Educação nutricional: passado, presente, futuro. Revista de Nutrição 1997; 10(1):5-19.

10. Valente FLS. Direito humano à alimentação: desafios e conquistas. São Paulo: Cortez; 2002. 272 p.

11. Boff L. Comer e beber juntos e viver em paz. Petrópolis: Vozes; 2006. 135 p.

12. Freire P. Educação como prática da liberdade. 30 ed. Rio de Janeiro: Paz e Terra; 2005. 157 p.

13. Vigotsky LS. A construção do pensamento e da linguagem. São Paulo: Martins Fontes; 2001. 520 p.

Recebido: 29/1/2015

Revisado: 10/3/2015

Aprovado: $31 / 3 / 2015$ 
